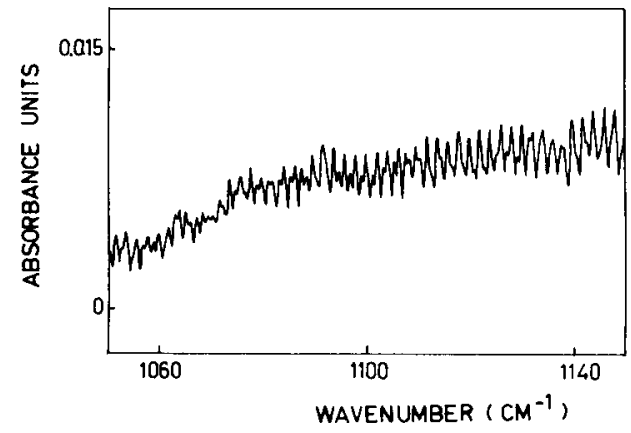

Fig. 9. Part of the infrared absorption spectrum where the $v_{3}$ vibration $\mathrm{CF}_{2}$ is located $\left(1102 \mathrm{~cm}^{-1}\right)$. The oscillations increase the detection limit to $10^{19}$ $\mathrm{m}^{-3}$.

by the relatively slow flow through the cuvette and that in contrast to that $\mathrm{CF}_{2}$ radicals are lost at the wall in the etching reaction the difference in density of two orders of magnitude can be understood.

\section{CONCLUSIONS}

Optical methods yield useful information from the complicated molecular plasma used in plasma etching. Emission spectra give the composition of the plasma rotational and vibrational temperatures, and densities of excited states when spectroscopic data are available. Infrared absorption gives absolute values of ground state densities of molecules and radicals. In our $\mathrm{CF}_{4}$ plasma we found the densities of the reaction products $\mathrm{SiF}_{4}$ and $\mathrm{CO}$ to be $2.5 \pm 0.4 \times 10^{20} \mathrm{~m}^{-3}$ and $3 \pm 2 \times 10^{20} \mathrm{~m}^{-3}$, respectively. The density of $\mathrm{CF}_{2}$ radicals could not be determined and is estimated to be below $10^{19} \mathrm{~m}^{-3}$.
The overall conclusion of our investigation is that the etching of quartz in a $\mathrm{CF}_{4}$ discharge takes place mainly through the reaction

$$
\mathrm{SiO}_{2}+2 \mathrm{CF}_{2} \rightarrow \mathrm{SiF}_{4}+2 \mathrm{CO} \text {. }
$$

\section{ACKNOWLEDGMENTS}

The authors are grateful for the many discussions with D.C. Schram, the critical remarks on this manuscript from F.J. de Hoog and the technical assistance by M.J.F. van de Sande.

\footnotetext{
a) Department of Electrical Engineering.

b) Physics Department.

${ }^{c)}$ Department of Chemical Engineering.

${ }^{1}$ W. R. Harshbarger and R. A. Porter, Solid State Technol. 21-4, 99 (1978).

${ }^{2}$ D. Field, A. J. Hydes, and D. F. Klemperer, Vacuum 34, 563 (1984).

${ }^{3}$ J. C. Knights, J. P. M. Schmitt, J. Perrin, and G. Guelachvili, J. Chem. Phys. 76, 342 (1982).

${ }^{4}$ H. U. Poll, D. Hinze, and H. Schlemann, Appl. Spectrosc. 36, 445 (1982).

${ }^{5} \mathrm{~J}$. Nishizawa and N. Hayasahi, Thin Solid Films 92, 189 (1982).

${ }^{6} \mathrm{~J}$. W. Coburn and M. Chen, J. Appl. Phys. 51, 3134 (1980).

${ }^{7}$ R. d'Agostino, V. Colaprico, and F. Cramarossa, Plasma Chem. Plasma Process. 1, 365 (1981).

${ }^{8}$ P. J. Hargis and M. J. Kushner, Appl. Phys. Lett. 40, 779 (1982).

${ }^{9}$ R. A. Gottscho, G. P. Davis, and R. H. Burton, Plasma Chem. Plasma Process. 3, 193 (1983).

${ }^{10}$ R. Bleekrode and W. van Benthem, J. Appl. Phys. 40, 5274 (1969).

"A. Medina and J. Morcillo, An. Fis. A 64, 251 (1968).

${ }^{12}$ P. Varanasi and S. Sarangi, J. Quant. Spectrosc. Radiat. Transfer 15, 473 (1975).

${ }^{13}$ W. B. Person and J. H. Newton, J. Mol. Struct. 46, 105 (1978)

${ }^{14}$ I. W. Levin and T. C. Lewis, J. Chem. Phys. 52, 1608 (1970).

${ }^{15}$ W. G. Golden, C. Marcott, and J. Overend, J. Chem. Phys. 68, 2081 (1978).

${ }^{16}$ J. W. Coburn and H. F. Winters, J. Vac. Sci. Technol. 16, 391 (1979).
}

\title{
An effective background removal technique for inelastic electron tunneling spectra
}

\author{
G. J. Gajda and W. H. Weinberg \\ Division of Chemistry and Chemical Engineering, California Institute of Technology, Pasadena, \\ California 91125
}

(Received 25 March 1985; accepted 28 June 1985)

This report describes a simple, rapid, and effective computer algorithm for background removal in inelastic electron tunneling spectra.

\section{INTRODUCTION}

Inelastic electron tunneling spectroscopy (IETS) is a highly sensitive surface vibrational spectroscopy of moderate resolution. As such, it has been applied to a wide variety of problems in surface science, and has been reviewed extensively. ${ }^{1,8}$ As in the case of all surface sensitive vibrational spectroscopies (e.g., IRAS, Raman, and EELS), IETS provides information on the molecular nature of the surface species. Unlike other vibrational spectroscopies, IETS has an intrinsically upward sloping background with increasing frequency. Several methods have been used to remove this background slope, including trial and error slope subtraction, ${ }^{9}$ Tchebycheff polynomials, ${ }^{9}$ and subtraction of one tunneling spectrum from another. ${ }^{10}$ Each of these methods has drawbacks due to excessive time of interactive trial and error methods, inaccuracy, or problems in matching spectra. In addition, some methods depend on a subjective determination of the "best" spectrum. This report describes a simple and rapid computer algorithm for the efficient, objective re- 


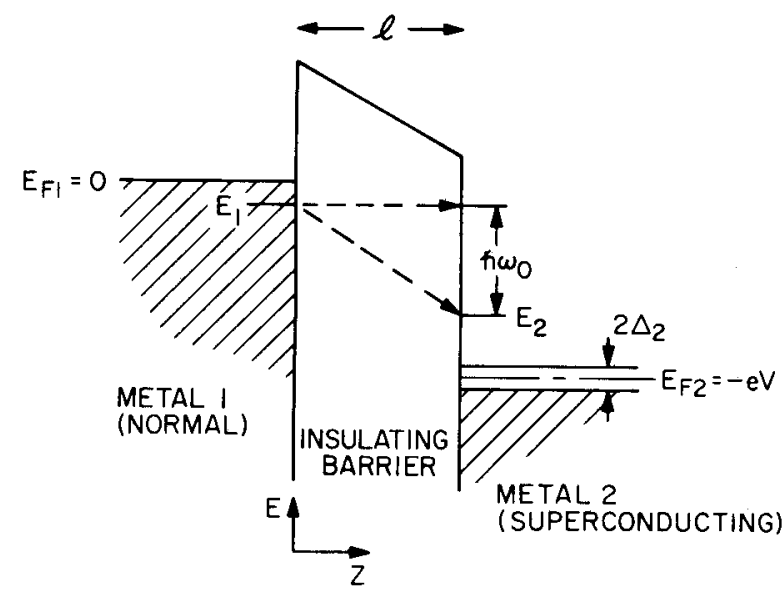

FIG. 1. Schematic energy diagram for the inelastic electron tunneling between energy level $E_{1}$ in a normal metal, and energy level $E_{2}$ in a superconducting metal of energy gap $2 \Delta_{2}$. The Fermi level of the normal metal is $E_{F 1}=0$, while that of the superconducting metal is $E_{F 2}=-\mathrm{eV}$, where eV is the applied bias voltage. Elastic tunneling is represented by the horizontal dashed line, whereas inelastic tunneling is represented by the oblique dashed line resulting in a vibrational excitation of energy $\hbar \omega_{0}$. The width of the insulating barrier is given by 1 .

moval of the background slope.

The upward sloping background characteristic of IET spectra is produced by the elastic tunneling which occurs in the junction. A schematic energy diagram of the tunnel junction is given in Fig. 1. As indicated, both elastic and inelastic tunneling can occur; the latter only if $\mathrm{eV}_{\text {bias }}$ is greater than $\hbar \omega_{0}$. Figure 2 schematically depicts the results of measuring the current $(I)$, conductance $[d I / d(\mathrm{eV})]$ or the second derivative of the current $\left[d^{2} I / d(\mathrm{eV})^{2}\right]$ as a function of bias voltage.
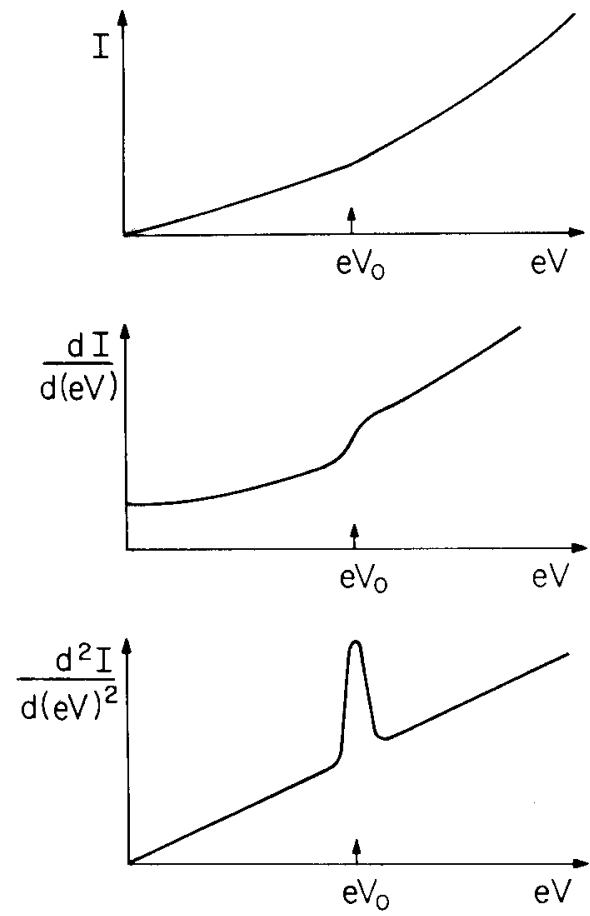

FIG. 2. Schematic representation of the current-voltage, the conductancevoltage and the second derivative of the current with respect to voltage for a tunneling junction. The latter is an "inelastic electron tunneling spectrum."
For small bias voltages $(\mathrm{eV}<0.5 \mathrm{~V})$, the elastic background is approximately given by

$$
I=\beta\left(V+\gamma V^{3}\right)
$$

where $\beta$ and $\gamma$ are functions of the barrier properties. ${ }^{11}$ This implies that

$$
\frac{d^{2} I}{d V^{2}}=6 \beta \gamma V
$$

or that the elastic background is a linear function of the bias voltage over the range of interest for vibrational data.

It should be noted that for IET spectra obtained by constant current systems, the background slope often decreases at high bias voltage $(\mathrm{eV} \sim 0.45-0.5 \mathrm{~V})$. This effect is not due to higher order terms in the expansion since all coefficients are positive and would thus predict an increasing slope. The flattening of the background is an artifact produced by measuring

$$
\frac{d^{2} V}{d I^{2}}=\frac{1}{\sigma^{3}} \frac{d^{2} I}{d V^{2}}
$$

For low resistance junctions, $\sigma_{500 \mathrm{mv}}-\sigma_{30 \mathrm{mV}}$ $\sim(0.1) \sigma_{30 \mathrm{mv}}$. Unless the second derivative spectra are corrected, errors of $(1.1)^{3}-1 \sim 30 \%$ can occur. A more complete discussion of errors and the necessary corrections is given by Adler. ${ }^{12}$ These methods, while devised for line shape analysis, can also be used to obtain background removed spectra. However, they require a measurement of the first harmonic spectrum as well as the second harmonic spectrum.

\section{DESCRIPTION}

The basic idea of any background correction routine in IETS is to calculate the average slope of the background. The quickest, and surprisingly accurate, method is to calculate the average first derivative of the spectrum. This is accomplished by using the least squares convolution method of Savitsky and Golay. ${ }^{13}$ A cubic derivative function 11 points

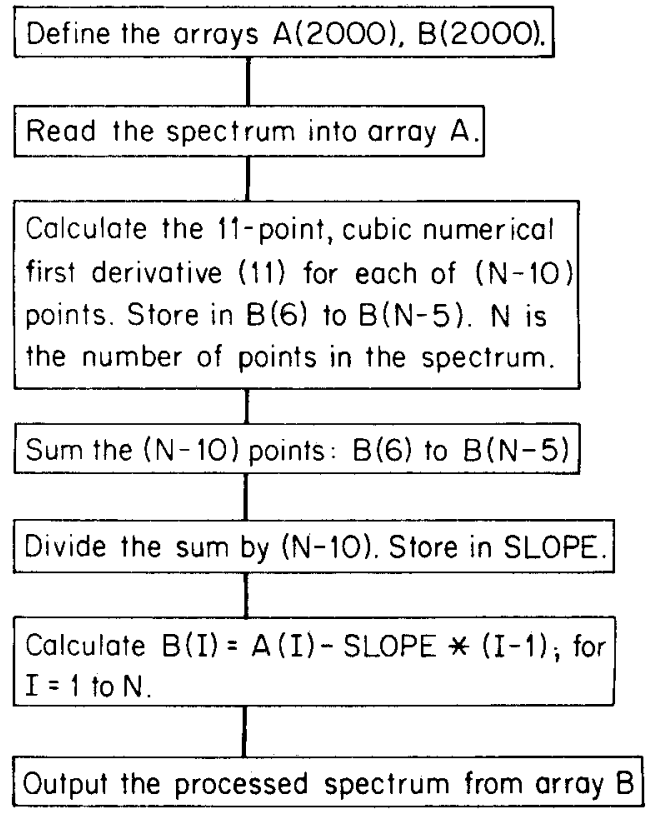

FIG. 3. Simplified flow chart of background slope removal subroutine. 


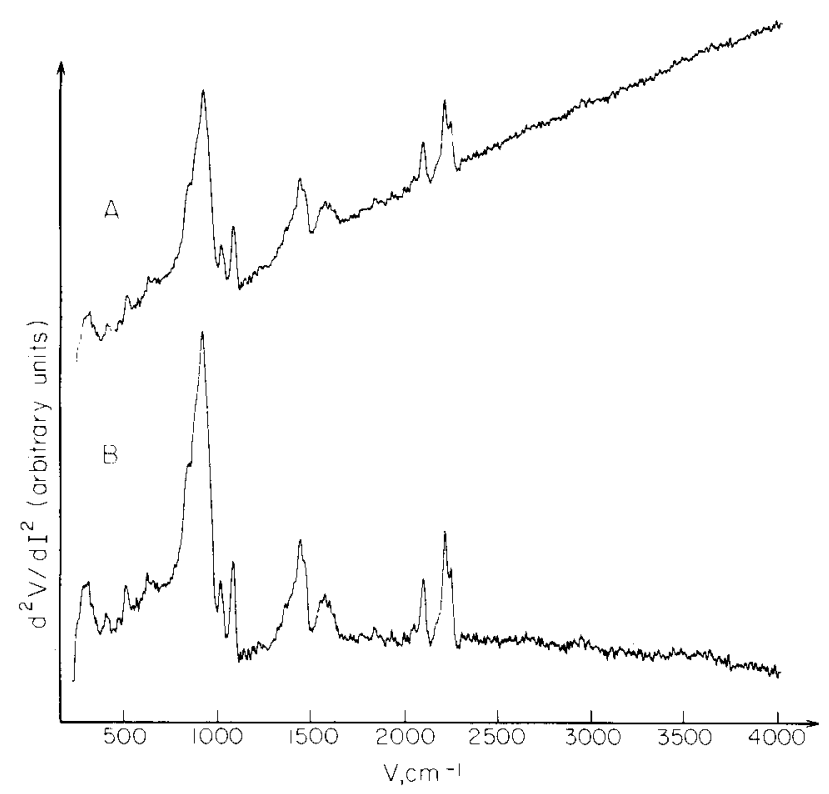

FIG. 4. Inelastic electron tunneling spectrum of $\mathrm{CD}_{3} \mathrm{COOD}$ adsorbed on $\mathrm{Al}_{2} \mathrm{O}_{3}$. [Lock-in time constant $\tau=1 \mathrm{~ms}, 3500$ scans.] (A) Raw data. (B) Data processed to remove background slope.

in length is used. The 11 points represent a compromise between smoothing spectra to minimize the effects of noise, and minimizing broadening to allow the first derivative to calculate peak positions, as described by Templeton. ${ }^{9}$ The values calculated at the $N-10$ fitting points are summed and then divided by $(N-10)$, where $N$ is the number of data points in the spectrum. The resulting value is the average slope and is the magnitude of a linear function which is then subtracted from the original data. This provides a flat background for the spectrum which facilitates comparison of the data with other spectra.

The routine easily handles large asymmetric peaks if the peaks start and end at the base line. Shoulders and poorly resolved peaks are also handled properly. The presence of a partial peak that occurs at the start of the spectrum $(240$ $\mathrm{cm}^{-1}$ ) will cause problems due to the slope of the peak not averaging to zero. The program checks to see if the spectrum starts at a value close to the minimum value in the entire spectrum, which indicates the absence of an interfering peak. If a peak occurs at $240 \mathrm{~cm}^{-1}$, it is skipped, and the correction routine starts at the minimum amplitude point in the spectrum. A flow chart of the routine employed is given in Fig. 3. The computer programs used to process the data are available upon request.

\section{RESULTS AND DISCUSSION}

As an example of this background removal technique, the results obtained from an $\mathrm{Al} / \mathrm{Al}_{2} \mathrm{O}_{3} / \mathrm{Pb}$ tunnel junction, the $\mathrm{Al}_{2} \mathrm{O}_{3}$ surface of which was exposed to $\mathrm{CD}_{3} \mathrm{COOD}$ from the vapor phase, are presented. The junction had a resistance of $992 \Omega$. The spectrum was obtained with the phase optimization technique described elsewhere. ${ }^{14}$ The raw data are presented in Fig. 4(A). This 965 point spectrum was then processed by the background removal routine, which required 4 s to execute on an LSI 11/23 microcomputer using FORTRAN IV with an RT-11SJ V4.0B operating system. The final spectrum is presented in Fig. 4(B).

The processed spectrum, with a relatively flat background, permits easier location of peak maxima, especially with a numerical derivative evaluation routine, ${ }^{9}$ and determination of "shoulders" on peaks. In addition, it removes the change in background values as the limiting factor in vertical spectrum expansion. [In Fig. 4(A), the spectrum amplitude is determined by the background value at 4000 $\mathrm{cm}^{-1}$. In Fig. 4(B), it is determined by the height of the $\mathrm{Al}-\mathrm{O}$ stretching mode at $940 \mathrm{~cm}^{-1}$.] This permits finer detail to be observed throughout the entire spectrum.

In summary, a rapid and simple computer algorithm for the removal for the sloping background from inelastic electron tunnel junction spectra has been presented. This algorithm allows easier evaluation of the data contained in the spectra, while providing an objective method of eliminating the sloping background in a single tunnel junction.

\section{ACKNOWLEDGMENT}

This work was supported by the Army Research Office under Grant No. DAAG29-83-K-0094.

${ }^{3}$ S. K. Khanna and J. Lambe, Science 220, 1345 (1983).

${ }^{2}$ Tunneling Spectroscopy, edited by P. K. Hansma (Plenum, New York, 1982).

${ }^{3}$ W. H. Weinberg, Vib. Spectra Struct. 11, 1 (1982).

${ }^{4}$ W. H. White and T. Wolfram, Methods Exp. Phys. A 16, 149 (1980).

${ }^{5}$ Inelastic Electron Tunneling Spectroscopy, edited by T. Wolfram (Springer, Berlin, 1978).

${ }^{6}$ W. H. Weinberg, Annu. Rev. Phys. Chem. 29, 115 (1978).

${ }^{7}$ P. K. Hansma and J. R. Kirtley, Acc. Chem. Res. 11, 440 (1978).

${ }^{8}$ P. K. Hansma, Phys. Rep. 30C, 146 (1977).

${ }^{9} \mathrm{M}$. K. Templeton, Ph. D. thesis, California Institute of Technology, 1984.

${ }^{10}$ W. M. Bowser and W. H. Weinberg, J. Am. Chem. Soc. 102, 4720 (1980).

${ }^{11}$ J. G. Simmons, J. Appl. Phys. 34, 238 (1963).

${ }^{12}$ J. G. Adler, in Tunneling Spectroscopy (Plenum, New York, 1982).

${ }^{13}$ A. Savitzky and M. J. E. Golay, Anal. Chem. 36, 1627 (1964).

${ }^{14}$ G. J. Gajda and W. H. Weinberg, Rev. Sci. Instrum. 56, 700 (1985). 